\title{
Portal venous gas and pneumatosis intestinalis
}

\author{
Shung-Wei Liu MD, Tai-Yu Huang MD
}

$\mathrm{A}$

60-year-old woman with a history of atrial fibrillation presented after one day of drowsiness and abdominal bloating. Examination revealed fever, a distended abdomen without muscle guarding and decreased bowel sounds. Results of blood tests and computed tomography (CT) scans of the head showed no specific abnormalities. A radiograph taken with the patient in the left lateral decubitus position to rule out acute abdomen showed branching radiolucency in the liver and gas in the bowel wall (Figure 1). Subsequent contrastenhanced CT scans of the abdomen revealed a left atrial thrombus, portal venous gas, a filling defect in the superior mesenteric artery and pneumatosis intestinalis (Appendix 1, available at www.cmaj.ca/lookup/suppl/doi:10.1503/cmaj $.120219 /-/ D C 1)$. Acute mesenteric infarction secondary to thromboembolism of cardiac origin was diagnosed. Because of septic shock, the patient died on the operating table before emergency surgery was started.

Portal venous gas and pneumatosis intestinalis, first described in 1955 and 1963 respectively, are radiographic signs that have been traditionally associated with bowel ischemia. ${ }^{1}$ Radiographic criteria for portal venous gas include a branching radiolucency that extends to within $2 \mathrm{~cm}$ of the liver capsule; this finding must be differentiated from that of pneumobilia (gas in the biliary system), which is visualized as air located centrally within the liver. ${ }^{1}$ Pneumatosis intestinalis is defined as the presence of gas within the bowel wall and is characterized by subserosal or submucosal gas-filled cysts in the gastrointestinal tract. Because of the widespread use of CT imaging, many nonischemic causes have been recently reported, including infectious, neoplastic and inflammatory bowel diseases for pneumatosis intestinalis, and portal pylephlebitis, abdominal trauma and iatrogenic procedures for portal venous gas. ${ }^{1,2}$ Therefore, neither of the radiographic signs of portal ve-

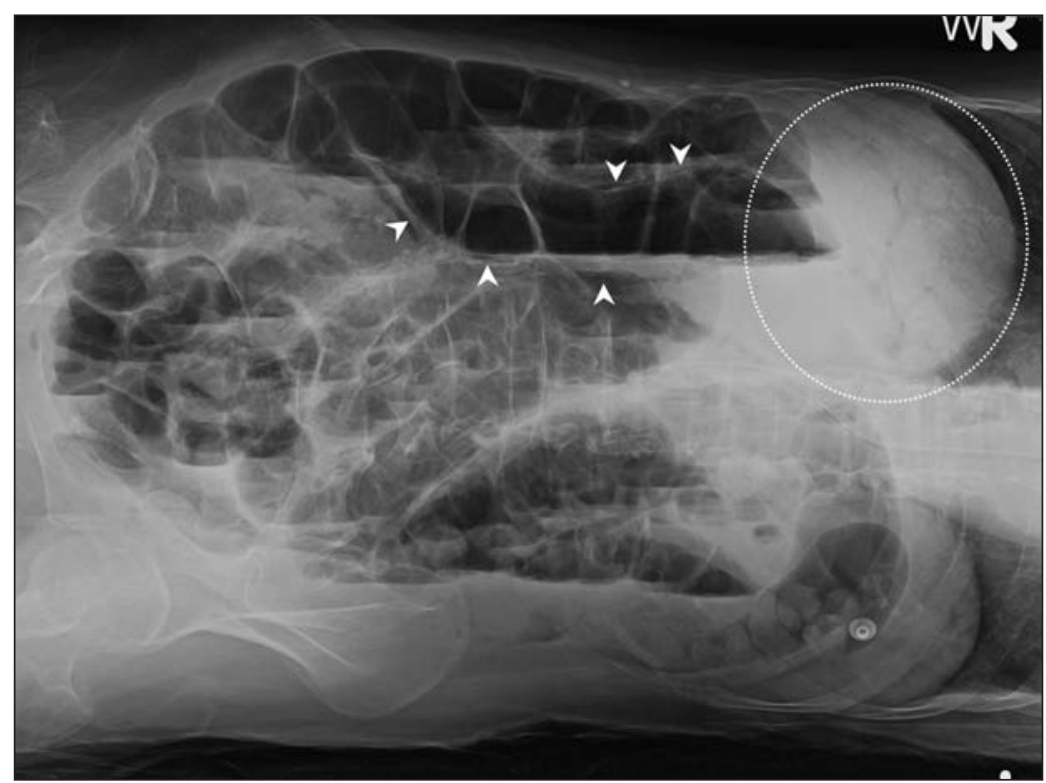

Figure 1: Radiograph of a 60-year-old woman with a distended abdomen taken in the left lateral decubitus position, showing branching air in the liver (circle) and gas in the bowel wall (arrowheads).

nous gas or pneumatosis intestinalis alone is definitive for diagnosing bowel ischemia. However, the simultaneous presence of both findings is more predictive of transmural bowel ischemia and higher mortality. ${ }^{1}$ If mesenteric ischemia is suspected, CT scanning is considered first-line imaging. ${ }^{2,3}$ However, appropriate interpretation of subtle findings on plain radiographs may help to make an early diagnosis of bowel ischemia. ${ }^{2,3}$

\section{References}

1. Wiesner W, Mortelé KJ, Glickman JN, et al. Pneumatosis intestinalis and portomesenteric venous gas in intestinal ischemia: correlation of CT findings with severity of ischemia and clinical outcome. AJR Am J Roentgenol 2001;177:1319-23.

2. Wayne E, Ough M, Wu A, et al. Management algorithm for pneumatosis intestinalis and portal venous gas: treatment and outcome of 88 consecutive cases. J Gastrointest Surg 2010;14:437-48.

3. Menke J. Diagnostic accuracy of multidetector CT in acute mesenteric ischemia: systematic review and meta-analysis. Radiology 2010;256:93-101.
Competing interests: None declared.

This article has been peer reviewed.

Affiliations: From the Department of Emergency Medicine, Buddhist Tzu Chi General Hospital, Hualien, Taiwan

Correspondence to:

Tai-Yu Huang, huangtaiyu72@gmail.com

CMAJ 2013. DOI:10.1503 /cmaj.120219 